\title{
Anti-inflammatory activity of palmitoylethanolamide ameliorates osteoarthritis induced by monosodium iodoacetate in Sprague-Dawley rats
}

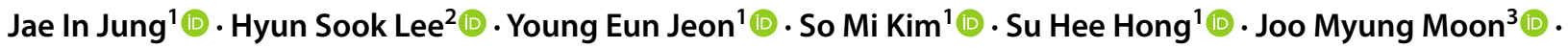 \\ Cho Young $\mathrm{Lim}^{3}$ (1) Yoon Hee Kim${ }^{3}$ (D) Eun Ji Kim ${ }^{1}$
}

Received: 15 June 2021 / Accepted: 19 August 2021 / Published online: 1 September 2021

(C) The Author(s) 2021

\begin{abstract}
Novel treatment strategies are urgently required for osteoarthritis (OA). Palmitoylethanolamide (PEA) is a naturally occurring fatty acid amide with analgesic and anti-inflammatory effects. We aimed to examine its effect on OA and elucidate the molecular mechanism of actions in monosodium iodoacetate (MIA)-induced OA Sprague-Dawley rats. The experimental animals were divided into normal control group (injected with saline + treated with phosphate-buffered saline (PBS), NOR), control group (injected with MIA + treated with PBS, CON), 50 or $100 \mathrm{mg} / \mathrm{kg}$ body weight (BW)/day PEA-treated group (injected with MIA + treated with 50 or $100 \mathrm{mg}$ of PEA/kg BW/day, PEA50 or PEA100), and positive control group (injected with MIA + treated with $6 \mathrm{mg}$ of diclofenac/kg BW/day, DiC). The changes in blood parameters, body parameters, gene expression of inflammatory mediators and cytokines, knee thickness, and joint tissue were observed. Oral administration of PEA had no adverse effects on the BW, liver, or kidneys. PEA reduced knee joint swelling and cartilage degradation in MIA-induced OA rats. The serum levels of leukotriene B4, nitric oxide, tumor necrosis factor (TNF)- $\alpha$, interleukin (IL)- $1 \beta$, and prostaglandin E2 considerably reduced in the PEA100 group compared with those in the CON group. In the synovia

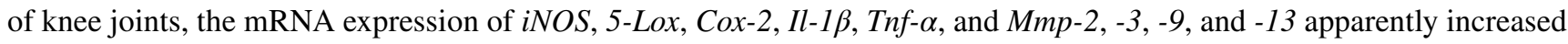
with MIA administration. Meanwhile, Timp-1 mRNA expression apparently decreased in the CON group but increased to the normal level with PEA treatment. Thus, PEA can be an effective therapeutic agent for OA.
\end{abstract}

Keywords Osteoarthritis $\cdot$ Palmitoylethanolamide (PEA) $\cdot$ Inflammation $\cdot$ Cytokine $\cdot$ Monosodium iodoacetate (MIA)

\section{Introduction}

Osteoarthritis $(\mathrm{OA})$ is a common progressive and degenerative joint disease that arises from joint cartilage destruction and joint tissue repair induced by inflammatory mediators (Alshami 2014; Steels et al. 2019). OA results from the

Jae In Jung, Hyun Sook Lee contributed equally to this study.

Eun Ji Kim

myej4@hallym.ac.kr

1 Regional Strategic Industry Innovation Center, Hallym University, 1 Hallymdaehak-gil, Chuncheon, Gangwon 24252, Republic of Korea

2 Department of Food Science and Nutrition, Dongseo University, Busan 47011, Republic of Korea

3 Technology Development Center, BTC Corporation, Ansan, Gyeonggi 15588, Republic of Korea failure of chondrocytes to control the balance between the synthesis and degradation of extracellular matrix components in articular cartilage (Man and Mologhianu 2014). The symptoms of OA include subchondral bone thickening, synovial inflammation, osteophyte formation, joint capsule hypertrophy, and ligament degeneration (Chen et al. 2017). These symptoms eventually lead to pain, stiffness, and disability, as well as lower the quality of life of the patients (Zhang et al. 2016).

As the cause of OA is unclear, its management is limited to medications to relieve pain, exercise therapy, weight control, prevention of complications such as muscle degeneration and joint deformity, and maintenance of the range of motion of the joint (Mora et al. 2018). Recently, it was reported that neuroinflammation caused by an imbalance between pro-inflammatory and pro-resolving mediators plays an important role in pain progression (Paladini et al. 2016). Chronic decline in immunity and increase in oxidative stress 
due to a natural immune response is observed in patients with OA (Chen et al. 2017; Mora et al. 2018). However, arthralgia is not always proportional to the degree of joint damage and immunity (Hochman et al. 2010). Long-term medications can alleviate OA pain but not cure OA (Bjordal et al. 2007).

Currently, the most commonly used OA pain relievers are non-steroidal anti-inflammatory drugs (NSAIDs), which are not very effective in relieving OA pain and exert adverse effects such as bleeding and gastric ulceration when used for a long period (Hinz and Brune 2004). Therefore, it is necessary to identify more effective and safe treatment strategies (Britti et al. 2017).

Palmitoylethanolamide (PEA), a type of $\mathrm{N}$-acylethanolamine (NAE), belongs to a family of endogenous bioactive lipids and is known to be effective against pain and inflammation (Re et al. 2007). PEA is a potential nutraceutical and a naturally occurring fat-derived signaling compound in many animals and plant foods, as well as in animal cells and tissues. The main food sources of PEA are soy lecithin, soybean, peanut, and corn (Petrosino and Di Marzo 2017). Generally, PEAs are manufactured by reacting palmitic acid and ethanolamine (LoVerme et al. 2005). Ethanolamine is usually derived from fossil fuels, but can also be manufactured through the decarboxylation reaction of serine (Scott et al. 2007). The decarboxylation reaction can be performed using pyridoxal 5'-phosphate-dependent serine decarboxylase, and this enzyme can be isolated from various plants such as spinach (Rontein et al. 2001). Among NAEs, PEA has been the most studied modulator of pain and inflammation (Steels et al. 2019). It has been shown to exert analgesic and anti-inflammatory effects in cells during inflammatory reactions and neurodegenerative conditions (Darmani et al. 2005). It has been reported that PEA increases in the spinal cord of animals with experimentally induced OA, and the PEA concentration decreases in the synovial fluid of patients with OA (Sagar et al. 2010; Richardson et al. 2008). In other words, PEA metabolism occurs abnormally in these diseases, and it is speculated that PEA supplementation may be helpful in treating these diseases or reducing their symptoms. The analgesic effect of PEA has also been reported in two cohort studies of patients with diseases such as OA (Gatti et al. 2012; Hesselink and Hekker 2012). In addition, in a recent study, PEA alleviated pain in patients with knee OA (Steels et al. 2019). However, the effect of PEA on joint disease-related pain is not fully understood, and more studies are needed to confirm the molecular mechanism of PEA action (Britti et al. 2017).

Monosodium iodoacetate (MIA)-induced OA Sprague-Dawley rats have been used as an OA experimental model. To experimentally induce OA in animals, carrageenan has been injected to cause acute and severe inflammation and pain. However, it has been reported that intra-articular injection of MIA is more likely to induce $\mathrm{OA}$ and the resulting inflammatory pain (D'Souza et al. 2011; Kuyinu et al. 2016). MIA induces chondrocyte cell death and bone lesions by interfering with a key glycolytic enzyme. MIA induction in the OA model increases inflammatory cytokines and causes pain, which gradually leads to nerve cell damage, nervous irritability, and neuralgia (Kuyinu et al. 2016). In this study, we aimed to determine the effect of PEA supplementation and its molecular mechanism of action in an experimental OA animal model.

\section{Materials and methods}

\section{Materials}

PEA (Levagen ${ }^{\circledR}$ ) was provided by Gencor Pacific Ltd, Hong Kong. Diclofenac sodium salt, MIA, 4',6-diamidino2-phenylindole (DAPI), and hematoxylin and eosin (H\&E) were purchased from Sigma-Aldrich Co. (St. Louis, MO, USA). Enzyme-linked immunosorbent assay (ELISA) kits for prostaglandin $\mathrm{E} 2\left(\mathrm{PGE}_{2}\right)$, leukotriene $\mathrm{B} 4\left(\mathrm{LTB}_{4}\right)$, tumor necrosis factor- $\alpha$ (TNF)- $\alpha$, and interleukin (IL)- $1 \beta$ were purchased from R\&D Systems (Minneapolis, MN, USA). Fluorochrome-conjugated secondary antibodies (Alexa 488 and 564) were purchased from Thermo Fisher Scientific (Waltham, MA, USA). Safranin O was purchased from ScienCell Research Laboratories (Carlsbad, CA, USA), and anti-aggrecan and anti-collagen type II alpha 1 (COL2A1) antibodies were purchased from Santa Cruz Biotechnology (Santa Cruz, CA, USA).

\section{Animals}

All experiments using animals reported herein were performed according to protocols approved by the Institutional Animal Care and Use Committee of Hallym University (HallymR1 2018-75). Six-week-old male Sprague-Dawley (SD) rats were purchased from DooYeol Biotech (Seoul, Korea). The rats were housed under specific pathogen-free conditions with a temperature of $23 \pm 3{ }^{\circ} \mathrm{C}$, relative humidity of $50 \% \pm 10 \%, 10-15$ times ventilation, $150-200 \mathrm{~lx}$ illumination, and a 12-h light/dark cycle. The rats were fed a commercial non-purified rodent diet (Cargill Agri Purina, Seongnam, Republic of Korea) with free access to water.

\section{Induction of MIA-induced $O A$ in rats}

After acclimation for 1 week, MIA-induced OA rat models were generated as described previously (Udo et al. 2016). The rats were anesthetized with $2-3 \%$ isoflurane $/ \mathrm{N}_{2} \mathrm{O} / \mathrm{O}_{2}$ mixture vapor using an inhalation anesthesia apparatus and ventilator (Fluovac System; Harvard Apparatus, Holliston, 
MA, USA). After anesthetization, $3 \mathrm{mg}$ of MIA in $50 \mu \mathrm{L}$ of saline was injected into the intra-articular joint of the right knee in all rats except those in the normal control group. Rats in the control group received an injection of saline instead of the MIA solution. One day after the injection of MIA, all rats were randomly divided into five groups: (1) normal control group (injected with saline + treated with phosphate-buffered saline (PBS) termed NOR, $n=10$ ); (2) control group (injected with MIA + treated with PBS, termed CON, $n=10$ ), (3) $50 \mathrm{mg} / \mathrm{kg}$ body weight (BW)/ day PEA-treated group (injected with MIA + treated with $50 \mathrm{mg}$ of PEA/kg BW/day termed PEA50, $n=10$ ); (4) $100 \mathrm{mg} / \mathrm{kg} \mathrm{BW} /$ day PEA-treated group (injected with $\mathrm{MIA}+$ treated with $100 \mathrm{mg}$ of PEA/kg BW/day termed PEA100, $n=10$ ), and (5) positive control group (injected with MIA + treated with $6 \mathrm{mg}$ of diclofenac/ $\mathrm{kg}$ BW/day termed DiC, $n=10$ ). The rats in each group were orally administered PEA or diclofenac once daily for 4 weeks. At the end of the experiment, isoflurane was used to anesthetize the animals and the thickness of both knees was measured with a digital caliper, and then blood samples were collected from the heart. The rats were sacrificed by carbon dioxide asphyxiation, and the knee joints and synovia were extracted for further analysis.

\section{Biochemical analyses of serum}

The serum levels of total cholesterol, triglyceride, glucose, blood urea nitrogen (BUN), and activities of aspartate aminotransferase (AST) and alanine aminotransferase (ALT) were measured using a blood chemistry autoanalyzer (KoneLab 20XT; Thermo Fisher Scientific, Vantaa, Finland).

\section{Micro-computed tomography (micro-CT) analysis}

To evaluate the changes in the knee joint microarchitecture, the femorotibial joint was scanned using a micro-CT scanner (VivaCT 80; Scanco Medical AG, Brüttisellen, Switzerland) with a source voltage of $70 \mathrm{keV}$, current of $114 \mu \mathrm{A}$, and isotropic resolution of $20 \mu \mathrm{m}$ at the Chuncheon Center of the Korea Basic Science Institute. Scans were integrated into three-dimensional images, and the three-dimensional morphometric parameters were calculated using micro-CT scanner image analysis software provided with the microCT scanner. The bone surface/bone volume (BS/BV, \%) of the subchondral bone in the femorotibial joint was analyzed to evaluate the degree of bone erosion. The bone volume fraction (bone volume/total volume, BV/TV, \%), trabecular thickness (Tb.Th, mm), and trabecular number (Tb.N, 1/ $\mathrm{mm}$ ) of the metaphysis of the tibia were analyzed to evaluate structural changes caused by various treatments.

\section{Histological analysis}

The knee joints were fixed with $4 \%$ paraformaldehyde, decalcified, embedded in paraffin, and cut into 5- $\mu \mathrm{m}$-thick sections. The sectioned tissues were stained with safranin $\mathrm{O}$ and H\&E. A light microscope (Axio Imager; Carl Zeiss Meditec AG, Jena, Germany) was used to observe the stained tissues. The randomly selected fields of the slides were photographed and examined in a blinded manner. The severity of knee joint OA was evaluated according to the Osteoarthritis Research Society International (OARSI) (Pritzker et al. 2006; Glasson et al. 2010).

\section{Measurement of nitric oxide (NO)}

As an indicator of NO production in the sera, the nitrite concentration in the sera was determined using the Griess Reagent System (Promega, Madison, WI, USA) in accordance with the manufacturer's instructions.

\section{ELISA}

The levels of PGE $2, \mathrm{LTB}_{4}, \mathrm{TNF}-\alpha$, and IL- $1 \beta$ in sera were measured using ELISA kits according to the manufacturer's instructions.

\section{Immunofluorescence (IF) staining}

Paraffin-embedded knee joint tissues were sectioned to a thickness of $5 \mu \mathrm{m}$, deparaffinized, and blocked using $5 \%$ bovine serum albumin. The indicated antibodies and fluorochrome-conjugated secondary antibodies (Alexa 488 or 564) were used to perform IF staining. DAPI was used to counterstain the nuclei. The randomly selected fields of the slides were photographed at $\times 400$ magnification and examined in a blinded manner. The Axio Imager microscope and AxioVision software (Carl Zeiss) were used to quantify the immune-positive cells.

\section{Quantitative real-time reverse transcription-polymerase chain reaction (RT-PCR)}

The total RNA was extracted from the synovia of the knee joints using TRIzol Reagent (Invitrogen Life Technologies, Carlsbad, CA, USA) according to the manufacturer's instructions. A micro-volume UV-Vis Spectrophotometer (BioSpec-nano, Shimadzu, Kyoto, Japan) was used to determine the content and purity of the total RNA. The total RNA $(2 \mu \mathrm{g})$ was reverse transcribed into complementary single stranded DNA using the HyperScript RT Master Mix kit (GeneAll Biotechnology, Seoul, Korea). Real-time PCR was performed using the Rotor-Gene SYBR Green PCR Kit (Qiagen, Valencia, CA, USA) and Rotor-Gene 3000 
instrument (Corbett Research, Mortlake, Australia), according to the manufacturer's instructions. Table 1 shows the nucleic acid sequences of the primers used in this study. The results were analyzed using Rotor-Gene 6000 Series software (Corbett Research, version 6). The relative expression levels of target genes were normalized to those of glyceraldehyde 3-phosphate dehydrogenase (Gapdh).

\section{Statistical analysis}

All data are expressed as mean \pm standard error of the mean of at least three independent experiments. GraphPad Prism 5.0 (GraphPad Software, San Diego, CA, USA) was used for statistical analysis. Differences between groups were analyzed using Student's $t$ test or the one-way analysis of variance, and statistical significance was set at $P<0.05$.

\section{Results}

\section{Oral administration of PEA caused no adverse effects}

Initially, we measured the BW of the rats during the experimental period and conducted a blood biochemical analysis to determine whether PEA administration caused adverse effects in vivo. Intra-articular injection of MIA or oral administration of PEA did not affect BW gain, serum levels of glucose and total cholesterol, BUN, and ALT activity. Serum triglyceride levels considerably increased with intraarticular MIA injection, and this increase noticeably reduced with the administration of PEA at $100 \mathrm{mg} / \mathrm{kg} \mathrm{BW}$. The administration of PEA considerably reduced AST activity, which tended to increase with intra-articular MIA injection. On the contrary, the administration of diclofenac noticeably reduced BW gain and serum glucose levels, compared with those in the NOR and CON groups (Table 2). These results indicate that there were no overt adverse effects in PEAtreated rats.

\section{Mitigation of knee joint swelling by PEA}

The thickness of the knee joint was measured to examine whether PEA administration mitigated the swelling of the knee joint. There was no observable difference in the left knee joint thickness without MIA injection in any of the experimental groups. The right knee joint thickness of the CON group was considerably higher than that of the NOR group. The administration of PEA decreased the thickness of the knee joints compared with that in the CON group. In addition, the right/left knee joint thickness ratio substantially reduced in the PEA100 group compared with that in the CON group (Table 3 ).

\section{PEA ameliorated cartilage degradation}

Micro-CT scans with three-dimensional remodeling were applied to MIA-induced OA rats to validate the anti-osteoarthritic effect of PEA. Three- and two-dimensional images from micro-CT scans revealed erosion of the subchondral and irregular articular surfaces in the CON group. The formation of subchondral erosion and irregular articular surface decreased with PEA administration (Fig. 1A, B). To quantify the degree of bone erosion, the BS/BV of the subchondral bone in the femorotibial joint was analyzed. BS/BV in the CON group was substantially higher than that in the NOR group. PEA administration substantially decreased BS/BV compared with that in the CON group (Fig. 1C). Within the metaphysis, Tb.N, Tb.Th, and BV/TV considerably decreased in the CON group compared with those in the NOR group. PEA administration considerably increased $\mathrm{BV} / \mathrm{TV}$ and Tb.N compared with those in the CON group, but did not affect Tb.Th (Fig. 1D). These results indicate that PEA administration ameliorated MIA-induced cartilage degradation and joint structure deformation.
Table 1 Primer sequences used in this study

\begin{tabular}{lll}
\hline & Forward primer $\left(5^{\prime}-3^{\prime}\right)$ & Reverse primer $\left(5^{\prime}-3^{\prime}\right)$ \\
\hline iNos & CACCACCCTCCTTGTCAAC & CAATCCACAACTCGCTCCAA \\
Cox -2 & TGCGATGCTCTT CCGAGCTGTGCT & TCAGGAAGTTCCTTATTTCCTTC \\
$5-$ Lox & CCATCCAGCTCAACCAAACC & GATGTGTGCGGAGAAGATGG \\
Tnf- $\alpha$ & AAATGGGCTCCCTCTCATCAGTTC & TCTGCTTGGTGGTTTGCTACGAC \\
$I l-1 \beta$ & CACCTCTCAAGCAGAGCACAG & GGGTTCCATGGTGAAGTCAAC \\
Mmp -2 & TGGGGGAGATTCTCACTTTG & CCATCAGCGTTCCCATACTT \\
Mmp -3 & TGGGAAGCCAGTGGAAATG & CCATGCAATGGGTAGGATGAG \\
Mmp -9 & TGCTCCTGGCTCTAGGCTAC & TTGGAGGTTTTCAGGTCTCG \\
Mmp-13 & TGGCGACAAAGTAGATGCTG & TGGCATGACTCTCACAATGC \\
Timp -1 & CTGAGAAGGGCTACCAGAGC & GTCATCGAGACCCCAAGGTA \\
Gapdh & CTCAACTACATGGTCTACATGTTCCA & CTTCCCATTCTCAGCCTTGACT \\
\hline
\end{tabular}


Table 2 Effect of PEA on body weight gain and blood biochemistry index in MIAinduced $\mathrm{OA}$ rats

\begin{tabular}{lccccc}
\hline & NOR & CON & PEA50 & PEA100 & DiC \\
\hline Initial body weight (g) & $180.9 \pm 2.9$ & $180.7 \pm 2.8$ & $180.9 \pm 1.6$ & $180.9 \pm 3.2$ & $180.7 \pm 1.1$ \\
Final body weight (g) & $376.3 \pm 7.3$ & $372.0 \pm 4.3$ & $365.7 \pm 5.4$ & $360.0 \pm 6.3$ & $355.7 \pm 5.1^{\#}$ \\
Body weight gain (g) & $195.4 \pm 7.0$ & $191.4 \pm 5.3$ & $184.8 \pm 6.1$ & $179.1 \pm 6.4$ & $175.0 \pm 5.2^{\#}$ \\
Glucose (mg/dL) & $168.1 \pm 11.7$ & $182.9 \pm 12.6$ & $177.9 \pm 11.9$ & $167.7 \pm 5.0$ & $142.7 \pm 9.2^{\#}$ \\
Triglyceride (mg/dL) & $62.5 \pm 4.8$ & $105.0 \pm 7.2^{* * *}$ & $87.6 \pm 6.6$ & $83.7 \pm 5.8^{\#}$ & $92.6 \pm 8.7$ \\
Total cholesterol (mg/dL) & $58.3 \pm 1.1$ & $60.4 \pm 2.6$ & $57.1 \pm 1.4$ & $60.2 \pm 1.4$ & $61.1 \pm 1.9$ \\
BUN (mg/dL) & $11.7 \pm 0.6$ & $13.1 \pm 0.4$ & $13.9 \pm 0.4$ & $14.0 \pm 0.6$ & $13.7 \pm 0.5$ \\
AST (U/L) & $120.0 \pm 15.3$ & $143.9 \pm 13.6$ & $94.6 \pm 4.2^{\# \#}$ & $103.1 \pm 7.3^{\#}$ & $105.7 \pm 6.9^{\#}$ \\
ALT (U/L) & $38.6 \pm 3.1$ & $42.3 \pm 3.7$ & $38.8 \pm 2.1$ & $36.7 \pm 1.3$ & $38.0 \pm 6.9$ \\
\hline
\end{tabular}

Values are expressed as mean $\pm \operatorname{SEM}(n=10){ }^{*} P<0.05,{ }^{* *} P<0.01,{ }^{* * *} P<0.001$ significantly different from the NOR group. ${ }^{\#} P<0.05,{ }^{\# \#} P<0.01,{ }^{\# \# \#} P<0.001$ significantly different from the CON group

$B U N$ blood urea nitrogen, $A S T$ aspartate aminotransferase, $A L T$ alanine aminotransferase, $P E A$ palmitoylethanolamide, $O A$ osteoarthritis, $M I A$ monosodium iodoacetate, $N O R$ normal control group (injected with saline + treated with phosphate-buffered saline (PBS)), CON control group (injected with MIA + treated with PBS), PEA50 or PEA100 50 or $100 \mathrm{mg} / \mathrm{kg}$ body weight (BW)/day PEA-treated group (injected with MIA + treated with 50 or $100 \mathrm{mg}$ of PEA/kg BW/day), DiC positive control group (injected with MIA + treated with $6 \mathrm{mg}$ of diclofenac/kg BW/day)

Table 3 Effect of PEA on knee thicknesses in MIA-induced OA rats

\begin{tabular}{lrrrrr}
\hline & NOR & CON & \multicolumn{1}{c}{ PEA50 } & PEA100 & DiC \\
\hline Left (normal) knee thicknesses (mm) & $10.56 \pm 0.14$ & $10.60 \pm 0.07$ & $10.41 \pm 0.14$ & $10.54 \pm 0.12$ & $10.28 \pm 0.03$ \\
Right (OA) knee thicknesses (mm) & $10.47 \pm 0.07$ & $12.37 \pm 0.11^{* * *}$ & $11.88 \pm 0.24$ & $11.92 \pm 0.14^{\#}$ & $11.19 \pm 0.11^{\# \#}$ \\
Right knee/left knee ratio & $1.00 \pm 0.01$ & $1.17 \pm 0.01^{* * *}$ & $1.14 \pm 0.02$ & $1.13 \pm 0.01^{\#}$ & $1.09 \pm 0.01^{\# \#}$ \\
\hline
\end{tabular}

Values are expressed as mean $\pm \operatorname{SEM}(n=10) .{ }^{*} P<0.05,{ }^{* *} P<0.01,{ }^{* * *} P<0.001$ significantly different from the NOR group. ${ }^{\#} P<0.05$, ${ }^{\# \#} P<0.01,{ }^{\# \# \# P} P<0.001$ significantly different from the CON group

PEA palmitoylethanolamide, $O A$ osteoarthritis, MIA monosodium iodoacetate, $N O R$ normal control group (injected with saline + treated with phosphate-buffered saline (PBS)), CON control group (injected with MIA + treated with PBS), PEA50 or PEA100 50 or $100 \mathrm{mg} / \mathrm{kg}$ body weight (BW)/day PEA-treated group (injected with MIA + treated with 50 or $100 \mathrm{mg}$ of PEA/kg BW/day), DiC positive control group (injected with MIA + treated with $6 \mathrm{mg}$ of diclofenac/kg BW/day)

In addition, we stained the knee cartilage with $\mathrm{H} \& \mathrm{E}$ and Safranin $\mathrm{O}$ to determine whether the administration of PEA alleviated the histopathology of OA in the knee joint. H\&E staining revealed well-preserved articular cartilage in the NOR group. However, the CON group exhibited severely damaged articular cartilage, as evidenced by the abraded surface. Safranin O staining results revealed less stained areas in the CON group, which indicates degraded proteoglycan in the extracellular matrix. The PEA50, PEA100, and DiC groups showed smoother articular surfaces and higher proteoglycan volumes than the CON group (Fig. 2). These results indicate that PEA administration reversed the histopathological changes induced by intra-articular MIA injection.

\section{PEA mitigated MIA-induced aggrecan and COL2A1 loss in articular cartilage}

IF staining results revealed that aggrecan expression considerably diminished in the articular cartilage of rats with
OA induced by intra-articular MIA injection. The PEA50, PEA100, and DiC groups presented higher aggrecan expression than the CON group (Fig. 3A). Similar to aggrecan expression in the articular cartilage, COL2A1 expression considerably decreased in the CON group compared with that in the NOR group. PEA administration substantially increased COL2A1 expression reduced by intra-articular MIA injection (Fig. 3B). The expression of aggrecan and COL2A1 increased 4.1- and 27.7-fold in the PEA100 group, respectively, compared with that in the $\mathrm{CON}$ group (Fig. 3C, D).

\section{PEA alleviated inflammation in MIA-induced $O A$ rats}

The serum levels of inflammatory mediators and cytokines were measured to investigate the effects of PEA on inflammation in MIA-induced OA rats. The ELISA results showed that the levels of NO, $\mathrm{PGE}_{2}, \mathrm{LTB}_{4}, \mathrm{TNF}-\alpha$, and IL-1 $\beta$ considerably increased in the sera of MIA-induced $\mathrm{OA}$ rats, and these changes substantially reduced with PEA 
Fig. 1 Effect of PEA on OA symptoms in MIA-induced OA rats. MIA-induced OA rats were administered either PEA $(50$ or $100 \mathrm{mg}$ / $\mathrm{kg} \mathrm{BW} /$ day) or diclofenac $(6 \mathrm{mg} / \mathrm{kg}$ BW/day) for 4 weeks. The femorotibial joint was scanned, integrated into three-dimensional microCT images, and analyzed with a micro-CT scanner and built-in software. a Three-dimensional micro-CT images. b Two-dimensional micro-CT images. c Gray scale reconstructed image for quantitative analysis and BS/BV (\%) of the subchondral bone in the femorotibial joint. d Gray scale reconstructed image for quantitative analysis, BV/TV (\%), Tb.N (1/mm), and Tb.Th $(\mathrm{mm})$ of the metaphysis of the tibia. Each bar represents mean \pm SEM $(n=5) .{ }^{*} P<0.01$, ${ }^{* *} P<0.05$, and ${ }^{* * *} P<0.001$ significantly different from the NOR group. ${ }^{\#} P<0.01,{ }^{\# \#} P<0.05$, and ${ }^{\# \# \#} P<0.001$ significantly different from the CON group. $P E A$ palmitoylethanolamide, $O A$ osteoarthritis, MIA monosodium iodoacetate, $B W$ body weight, NOR normal control group (injected with saline + treated with phosphate-buffered saline (PBS)), CON control group (injected with MIA + treated with PBS), PEA50 or PEA100 50 or $100 \mathrm{mg} / \mathrm{kg}$ body weight (BW)/day PEA-treated group (injected with MIA + treated with 50 or $100 \mathrm{mg}$ of PEA/kg BW/day), DiC positive control group (injected with MIA + treated with $6 \mathrm{mg}$ of diclofenac/kg BW/day), micro-CT micro-computed tomography, $B S / B V$ bone surface/bone volume, $B V / T V$ bone volume/total volume, $T b . N$ trabecular number, $T b . T h$ trabecular thickness

administration. The serum levels of NO, $\mathrm{PGE}_{2}, \mathrm{LTB}_{4}$, TNF$\alpha$, and IL- $1 \beta$ reduced by $57.8 \%, 14.7 \%, 43.7 \%, 22.7 \%$, and $21.8 \%$ in the PEA100 group, respectively, compared with those in the CON group (Table 4).

Next, we performed real-time RT-PCR to investigate the effects of PEA on the expression of inflammatory mediators and cytokines in the synovia of the knee joints. As shown in Fig. 4, the mRNA expression of iNos, 5-lipoxygenase (Lox), cyclooxygenase (Cox)-2, Tnf- $\alpha$, and Il-1 $\beta$ substantially increased in the synovia of MIA-induced OA rats, which was suppressed by the administration of PEA. In the PEA100 group, the mRNA expression of iNos, 5-Lox, Cox-2, Tnf- $\alpha$, and $\mathrm{Il}-1 \beta$ reduced by $41.8 \%, 66.4 \%, 28.9 \%, 64.6 \%$, and $53.8 \%$, respectively, compared with that in the CON group (Fig. 4).

\section{PEA modulated the expression of Mmp and Timp in the synovia of MIA-induced OA rats}

In the synovia of the knee joints, the mRNA expression of matrix metalloproteinase (Mmp)-2, Mmp-3, Mmp-9, and Mmp-13 noticeably increased with intra-articular MIA injection, and these changes substantially reduced with PEA administration at $100 \mathrm{mg} / \mathrm{kg}$ BW. Meanwhile, tissue inhibitor of metalloproteinase (Timp)-1 mRNA expression considerably reduced in the $\mathrm{CON}$ group, but this reduction was prevented by PEA administration at $100 \mathrm{mg} / \mathrm{kg}$ BW (Fig. 5). (a)
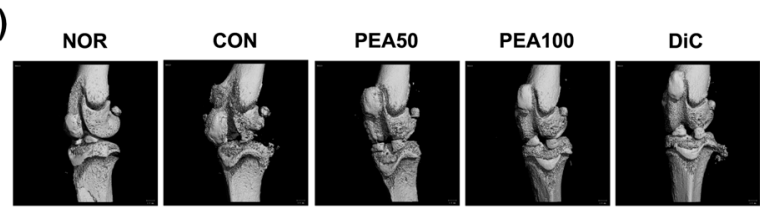

(b)
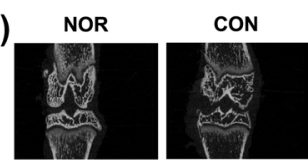

PEA50

PEA100
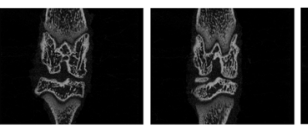

DiC

(c)
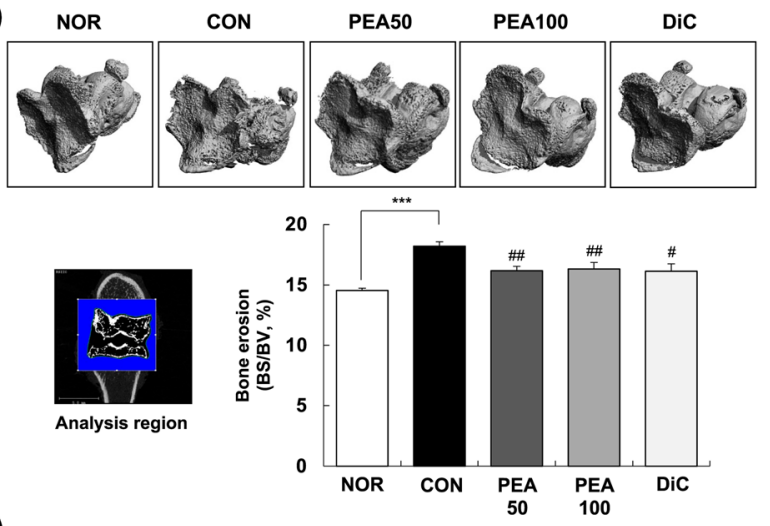

(d)

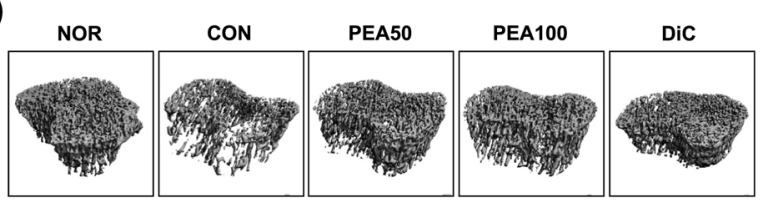

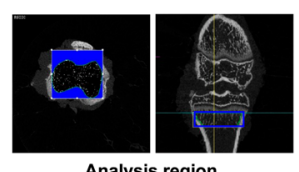

Analysis region

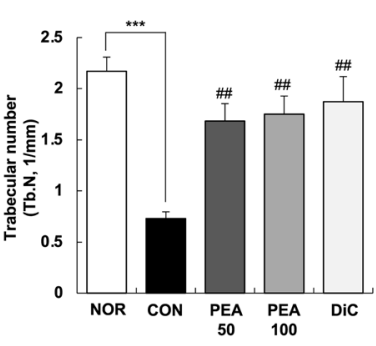

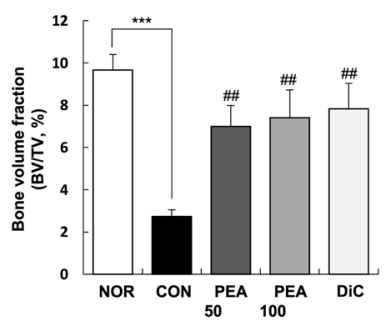

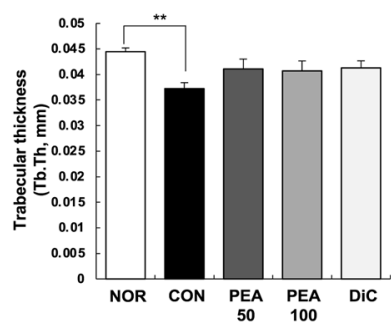

\section{Discussion}

We confirmed that PEA mitigated the symptoms of MIAinduced knee OA based on the following findings: (1) PEA reduced swelling of the knee joint (Table 3), (2) PEA ameliorated cartilage degradation and joint structure deformation (Fig. 1), (3) PEA reversed histopathological changes (Fig. 2), and (4) PEA mitigated aggrecan and COL2A1 loss in the articular cartilage (Fig. 3). PEA did not cause any adverse effects. PEA treatment alleviated 
Fig. 2 Effect of PEA on histological changes in the articular cartilage in MIA-induced OA rats. MIA-induced OA rats were administered either PEA (50 or $100 \mathrm{mg} / \mathrm{kg} \mathrm{BW/day)}$ or diclofenac $(6 \mathrm{mg} / \mathrm{kg} \mathrm{BW} /$ day) for 4 weeks. Articular cartilage was stained with $\mathrm{H} \& \mathrm{E}$ (a) and safranin $\mathrm{O}(\mathbf{b})$, Research Society International (OARSI) scoring (c). Representative staining images are shown. Scale bar, $50 \mu \mathrm{m}$. Each bar represents mean \pm SEM $(n=5) .{ }^{*} P<0.01,{ }^{* *} P<0.05$, and ${ }^{* * * *} P<0.001$ significantly different from the NOR group. ${ }^{\#} P<0.01,{ }^{\# \#} P<0.05$, and ent from the CON group. PEA palmitoylethanolamide, $O A$ iodoacetate, $B W$ body weight, $H \& E$ hematoxylin and eosin, NOR normal control group (injected with saline + treated (PBS)), CON control group (injected with MIA + treated with PBS), PEA50 or PEA100 50 or $100 \mathrm{mg} / \mathrm{kg}$ body weight (BW)/day PEA-treated group (injected with MIA + treated with 50 or $100 \mathrm{mg} / \mathrm{kg} \mathrm{BW/}$ day), $\mathrm{DiC}$ positive control group (injected with MIA + treated with $6 \mathrm{mg}$ of diclofenc/ $\mathrm{kg} \mathrm{BW/}$ day) and subjected to Osteoarthritis

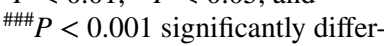
osteoarthritis, MIA monosodium with phosphate-buffered saline

(a)

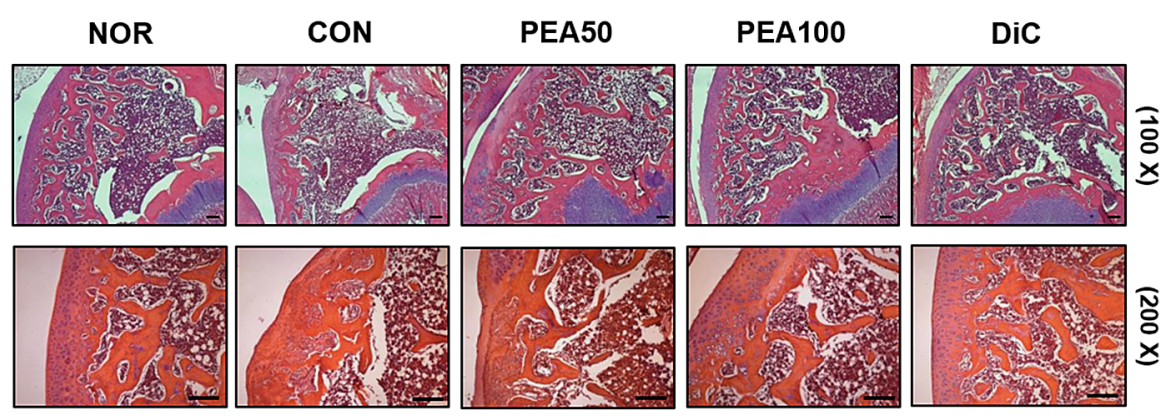

(b)
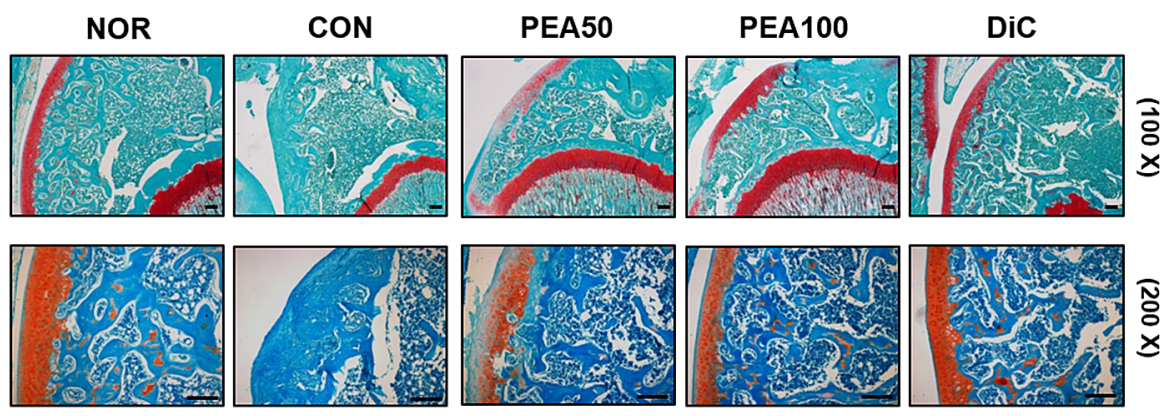

(c)

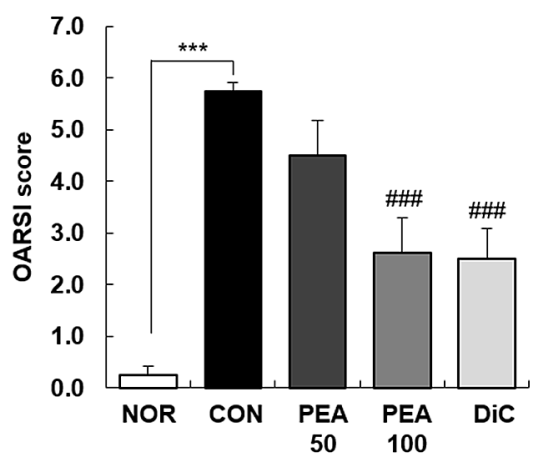

hypertriglyceridemia and increased AST levels in MIAinjected rats (Table 2). Another common adverse effect of arthritis medication is hypomotility (Alsalem et al. 2019). In our study, MIA-induced OA rats that were administered diclofenac, an arthritis drug, had substantially reduced physical activity throughout the day and did not respond to external stimuli. However, when PEA was administered, the physical activity of the rats considerably recovered (data not shown).

The main symptoms of OA are joint cartilage degeneration, chronic pain, and synovial inflammation (Saklatvala 1986). In our study, the right knees of rats that were administered MIA showed cartilage degradation and decreased bone volume fraction, trabecular number and thickness, and articular cartilage damage compared with the NOR group (Fig. 1 and 2). It is necessary to maintain a healthy synovial microenvironment to maintain cartilage integrity and homeostasis. The anti-hyperanalgesic effects of PEA involve inflammatory mediators, pro-inflammatory cytokines, neutrophil infiltration, and pro-inflammatory enzymes (i.e. COX-2 and iNOS), pro-inflammatory kinases (mitogen-activated protein kinase) (Costa et al. 2008), and neurotrophic factors (i.e. nerve growth factor), mast cell degranulation through autacoid local injury antagonism, histamine, and inhibition of $\mathrm{PGE}_{2}$ secretion (Costa et al. 2008; Alhouayek and Muccioli 2014).

The expression of these pro-inflammatory cytokines is higher in the joint tissue of patients with OA than in healthy subjects (Richardson et al. 2008). In our study, inflammatory mediators such as $\mathrm{NO}, \mathrm{PGE}_{2}$, and $\mathrm{LTB}_{4}$, and 
(a)

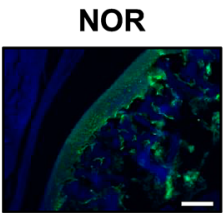

CON

PEA50

PEA100

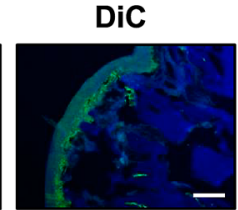

(b)
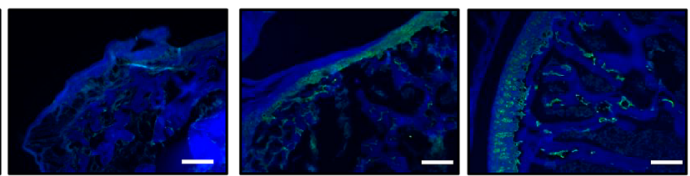

PEA100
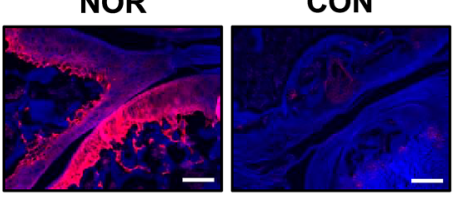

PEA50
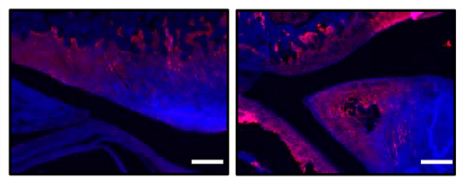

DiC

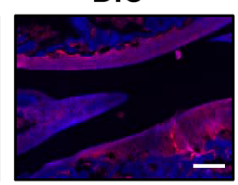

(c)

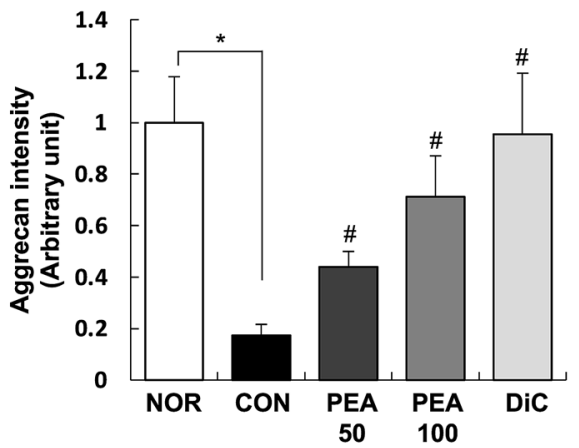

(d)

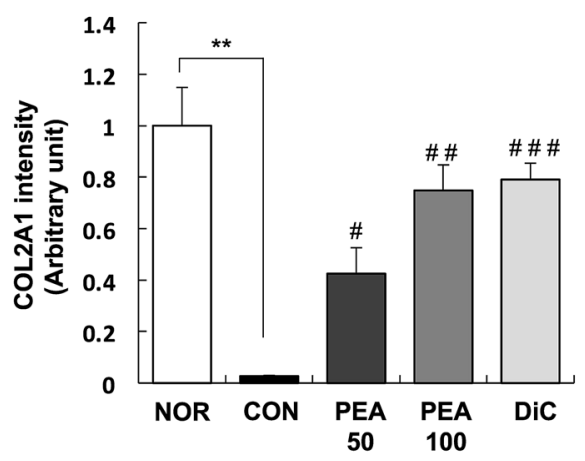

Fig. 3 Effect of PEA on the expression of aggrecan and COL2A1 in the articular cartilage of MIA-induced OA rats. MIA-induced OA rats were administered either PEA (50 or $100 \mathrm{mg} / \mathrm{kg} \mathrm{BW} /$ day) or diclofenac $(6 \mathrm{mg} / \mathrm{kg}$ BW/day) for 4 weeks. Articular cartilage was stained with aggrecan (a) and COL2A1 (b) antibodies. Representative IF staining images are shown. Scale bar, $50 \mu \mathrm{m}$. (c), (d) Staining intensity of the indicated proteins was quantified. Each bar represents mean \pm SEM $(n=5) .{ }^{*} P<0.01,{ }^{* *} P<0.05$, and ${ }^{* * * *} P<0.001$ significantly different from the NOR group. ${ }^{\#} P<0.01$, ${ }^{\# \#} P<0.05$, and ${ }^{\# \# \#} P<0.001$ significantly different from the CON group. COL $2 A 1$

collagen type II alpha 1, PEA palmitoylethanolamide, $I F$ immunofluorescence, $O A$ osteoarthritis, $M I A$ monosodium iodoacetate, $B W$ body weight, NOR normal control group (injected with saline + treated with phosphate-buffered saline (PBS)), CON control group (injected with MIA + treated with PBS), PEA50 or PEA100 50 or $100 \mathrm{mg} /$ $\mathrm{kg}$ body weight (BW)/day PEA-treated group (injected with MIA + treated with 50 or $100 \mathrm{mg}$ of PEA/kg BW/day), DiC positive control group (injected with MIA + treated with $6 \mathrm{mg}$ of diclofenac/kg BW/ day)

Table 4 Effect of PEA on serum levels of inflammatory mediators and cytokines in MIA-induced OA rats

\begin{tabular}{lrcccc}
\hline & \multicolumn{1}{l}{ NOR } & \multicolumn{1}{l}{ CON } & PEA50 & PEA100 & \multicolumn{1}{l}{ DiC } \\
\hline NO $(\mu \mathrm{M})$ & $5.75 \pm 0.48$ & $9.81 \pm 1.47^{*}$ & $4.33 \pm 0.29^{\# \#}$ & $4.14 \pm 0.26^{\# \#}$ & $4.55 \pm 0.24^{\# \#}$ \\
PGE $_{2}(\mathrm{ng} / \mathrm{mL})$ & $6.43 \pm 0.25$ & $7.33 \pm 0.18^{*}$ & $7.06 \pm 0.18$ & $6.25 \pm 0.32^{\#}$ & $5.13 \pm 0.54^{\# \#}$ \\
$\mathrm{LTB}_{4}(\mathrm{ng} / \mathrm{mL})$ & $1.34 \pm 0.08$ & $2.08 \pm 0.24^{*}$ & $1.26 \pm 0.14^{\#}$ & $1.17 \pm 0.08^{\# \#}$ & $1.23 \pm 0.14^{\# \#}$ \\
$\mathrm{TNF}-\alpha(\mathrm{pg} / \mathrm{mL})$ & $26.66 \pm 0.59$ & $32.67 \pm 1.73^{* *}$ & $25.64 \pm 0.49^{\# \#}$ & $25.24 \pm 0.65^{\# \#}$ & $26.76 \pm 0.63^{\# \#}$ \\
$\mathrm{IL}-1 \beta(\mathrm{pg} / \mathrm{mL})$ & $72.90 \pm 3.02$ & $83.88 \pm 3.36^{*}$ & $69.41 \pm 4.61^{\#}$ & $65.63 \pm 1.87^{\# \# \#}$ & $72.60 \pm 1.88^{\#}$ \\
\hline
\end{tabular}

Values are expressed as mean $\pm \operatorname{SEM}(n=10) .{ }^{*} P<0.05,{ }^{* *} P<0.01,{ }^{* * *} P<0.001$ significantly different from the NOR group. ${ }^{\#} P<0.05,{ }^{\# \#} P<0.01,{ }^{\# \#} P<0.001$ significantly different from the CON group

$O A$ osteoarthritis, $P E A$ palmitoylethanolamide, $M I A$ monosodium iodoacetate, $N O$ nitric oxide, $P G E_{2}$ prostaglandin E2, $L T B_{4}$ leukotriene B4, TNF- $\alpha$ tumor necrosis factor- $\alpha, I L-1 \beta$ interleukin- $1 \beta$, NOR normal control group (injected with saline + treated with phosphate-buffered saline (PBS)), CON control group (injected with MIA + treated with PBS), PEA50 or PEA100 50 or $100 \mathrm{mg} / \mathrm{kg}$ body weight (BW)/day PEAtreated group (injected with MIA + treated with 50 or $100 \mathrm{mg}$ of PEA/kg BW/day), DiC positive control group (injected with MIA + treated with $6 \mathrm{mg}$ of diclofenac/kg BW/day) 
Fig. 4 Effect of PEA on the expression of inflammatory mediators and cytokines in the synovia of MIA-induced OA rats. MIA-induced OA rats were administered either PEA (50 or $100 \mathrm{mg} / \mathrm{kg} \mathrm{BW/day)} \mathrm{or}$ diclofenac $(6 \mathrm{mg} / \mathrm{kg}$ BW/day) for 4 weeks. The total RNA was extracted from the synovia and reverse transcribed, followed by amplification using real-time PCR. Expression of target mRNA was normalized to that of GAPDH and represented relative to that of the NOR group. Each bar represents mean $\pm \operatorname{SEM}(n=10) .{ }^{*} P<0.01$,

${ }^{* *} P<0.05$, and ${ }^{* * *} P<0.001$ significantly different from the NOR group. ${ }^{\#} P<0.01$, ${ }^{\# \#} P<0.05$, and ${ }^{\# \# \#} P<0.001$ significantly different from the CON group. PEA palmitoylethanolamide, $O A$ osteoarthritis, MIA monosodium iodoacetate, iNos inducible nitric oxide synthase, Cox-2 cyclooxygenese-2, 5-Lox 5-lipoxygenase, Tnf- $\alpha$ tumor necrosis factor- $\alpha, I l-1 \beta$ interleukin-1 $\beta$, Gapdh glyceraldehyde 3-phosphate dehydrogenase, NOR normal control group (injected with saline + treated with phosphate-buffered saline (PBS)), CON control group (injected with MIA + treated with PBS), PEA50 or PEA100 50 or $100 \mathrm{mg} / \mathrm{kg}$ body weight (BW)/day PEA-treated group (injected with MIA + treated with 50 or $100 \mathrm{mg}$ of PEA $/ \mathrm{kg}$ BW/day), $\mathrm{DiC}$ positive control group (injected with MIA + treated with $6 \mathrm{mg}$ of diclofenac/ $\mathrm{kg} \mathrm{BW/day)}$

(a)

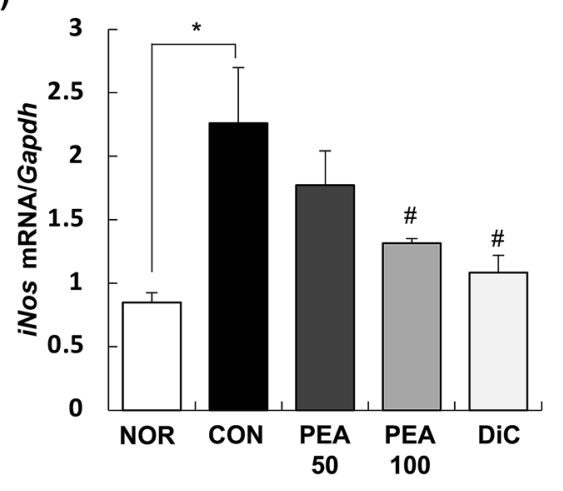

(b)

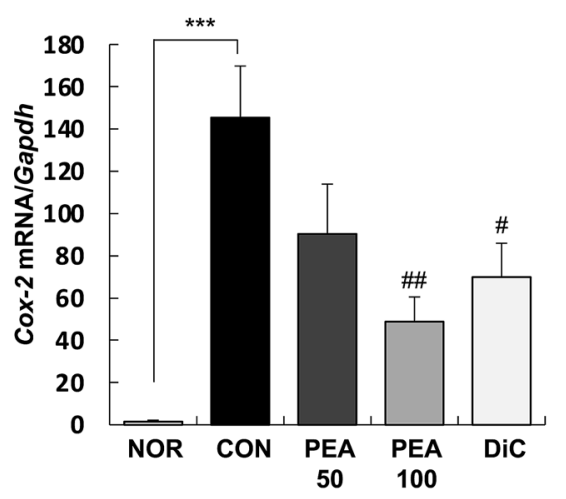

(d)

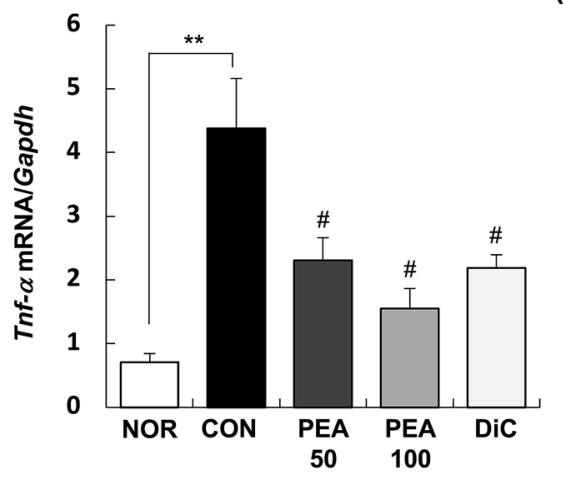

(c)

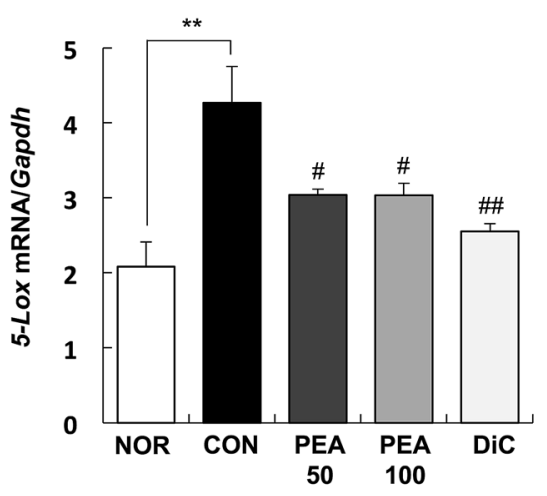

(e)

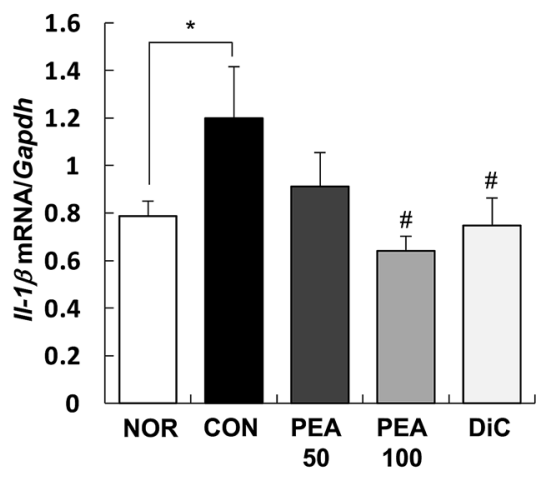

pro-inflammatory cytokines such as TNF- $\alpha$ and IL- $1 \beta$ considerably increased in the serum of the MIA-induced OA rats, but decreased to the same levels as those in the NOR group with PEA treatment (Table 4). In addition, the expression of iNos, 5-Lox, Cox-2, Tnf- $\alpha$, and Il-1 $\beta$ increased in the synovia of the knee joints with MIA treatment, but considerably decreased with PEA treatment (Fig. 4).

Inflammation occurs in joints via inflammatory mediators and pro-inflammatory cytokines, which in turn stimulate the secretion of cartilage-degrading enzymes such as MMP, thereby increasing cartilage degradation (Chadjichristos et al. 2003). We analyzed the mRNA expression of Mmp-2, Mmp-3, Mmp-9, Mmp-13, and Timp-1 in the synovia of the knee joints to determine whether PEA directly affects Mmp expression. The mRNA expression of all four types of MMPs increased substantially and that of TIMP-1 decreased with intra-articular MIA injection. These changes decreased in a dose-dependent manner and recovered to a similar degree as that of the DiC group when $100 \mathrm{mg} / \mathrm{kg} \mathrm{BW}$ PEA was administered (Fig. 5). In an experimental animal model of MIA-induced OA, PEA improved motor function and protected cartilage (Britti et al. 2017). PEA can relieve knee OA symptoms and protect the joints in patients with mild OA (Steels et al. 2019).

PEA is a cannabinoid-like compound, and the endocannabinoid system has emerged as a popular target for the development of new pain relievers (La Porta et al. 2014). Structurally, NAEs and PEA act via unique mechanisms 
Fig. 5 Effect of PEA on the expression of MMPs and TIMP-1 in the synovia of MIAinduced OA rats. MIA-induced $\mathrm{OA}$ rats were administered either PEA (50 or $100 \mathrm{mg} / \mathrm{kg}$ $\mathrm{BW} /$ day $)$ or diclofenac $(6 \mathrm{mg} / \mathrm{kg}$ BW/day) for 4 weeks. The total RNA was extracted from the synovia and reverse transcribed, followed by amplification using real-time PCR. Expression of target mRNA was normalized to that of Gapdh and represented relative to that of the NOR group. Each bar represents mean $\pm \operatorname{SEM}(n=10) .{ }^{*} P<0.01$, ${ }^{* *} P<0.05$, and ${ }^{* * *} P<0.001$ significantly different from the NOR group. ${ }^{\#} P<0.01$, ${ }^{\# \#} P<0.05$, and ${ }^{\# \# \#} P<0.001$ significantly different from the CON group. $P E A$ palmitoylethanolamide, $O A$ osteoarthritis, MIA monosodium iodoacetate, $M M P$ matrix metalloproteinase, TIMP-1 tissue inhibitor of metalloproteinase-1, NOR normal control group (injected with saline + treated with phosphate-buffered saline (PBS)), CON control group (injected with MIA + treated with PBS), PEA50 or PEA100 50 or $100 \mathrm{mg} / \mathrm{kg}$ body weight (BW)/day PEA-treated group (injected with MIA + treated with 50 or $100 \mathrm{mg}$ of PEA $/ \mathrm{kg}$ BW/day), DiC positive control group (injected with MIA + treated with $6 \mathrm{mg}$ of diclofenac/ $\mathrm{kg} \mathrm{BW/day)}$ (a)

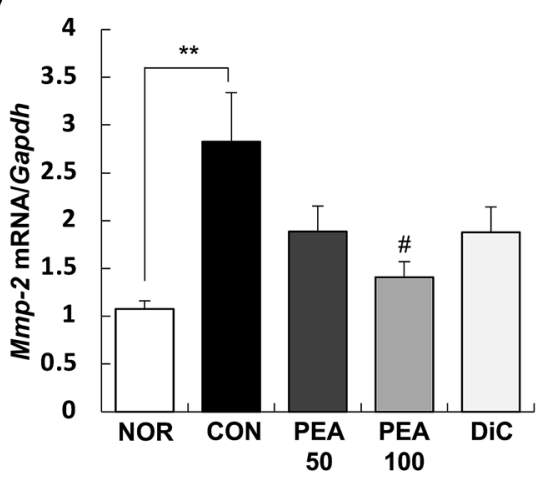

(c)

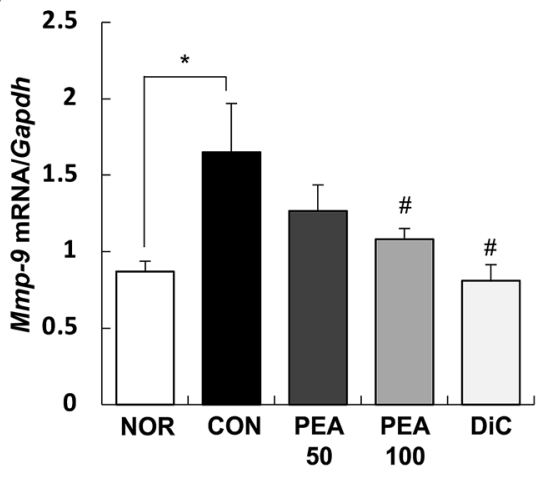

(b)

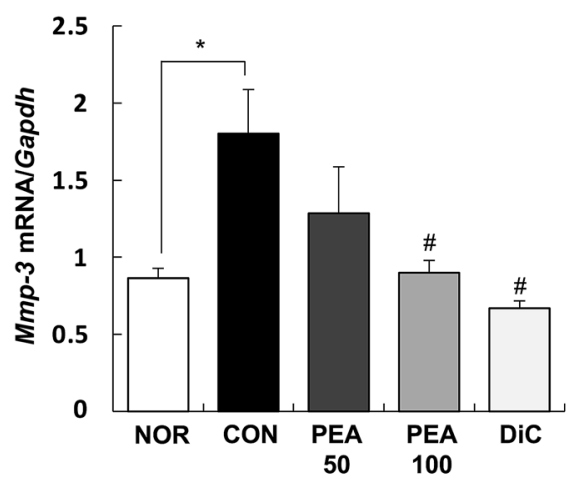

(d)

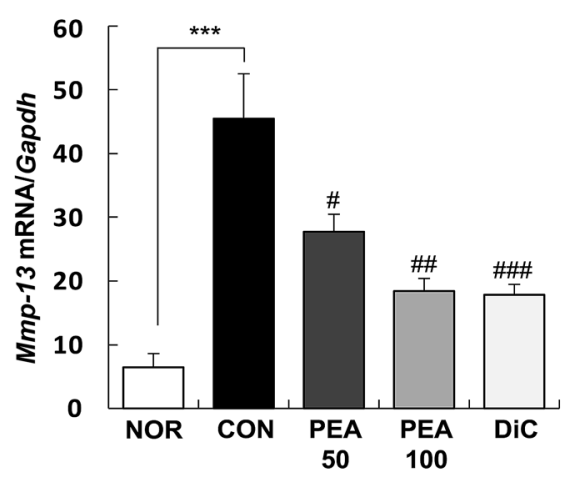

(e)

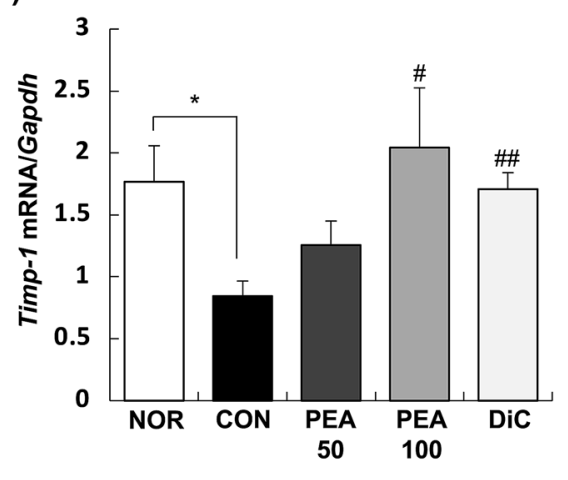

related to several biological pathways (Impellizzeri et al. 2013). The direct molecular targets of PEA are PPAR- $\alpha$ and GPR55, and the indirect molecular targets are transient receptor potential vanilloid type- 1 channel and cannabinoid-1 and -2 receptors (Petrosino and Di Marzo 2017; Guida et al. 2017). PEA directly or indirectly activates these molecular targets or receptors to exert its unique actions. In other words, when PEA activates PPAR- $\alpha$, PPAR- $\alpha$ reduces the expression of anti-inflammatory proteins such as IкB$\alpha$, which interferes with the translocation of nuclear factor (NF)- $\mathrm{KB}$ and inhibits the expression of the pro-inflammatory protein TNF- $\alpha$. Ultimately, the recruitment of immune cells is reduced (Alhouayek and Muccioli 2014). Regarding OA, PPAR- $\alpha$ is involved in inhibiting inflammation

and preventing the development of chondrogenic factors (Fahmi et al. 2001). PPAR- $\alpha$ decreases the expression of MMP-9 and TGF- $\beta$ (Wang et al. 2016). WY-14643, a potent PPAR- $\alpha$ agonist, inhibits the expression of metalloproteinases such as MMP-1, -3, and -13 in cartilage explants of human OA (Clockaerts et al. 2011). WY-14643 also exhibits anti-inflammatory effects by inhibiting pro-inflammatory factors such as NO, $\mathrm{PGE}_{2}$, MCP-1, IL-6, IL-1 $\beta$, and TNF- $\alpha$ through the NF- $\kappa B$ pathway (Huang et al. 2016). The anti-inflammatory effect of the PPAR- $\alpha$ receptor eventually leads to the inhibition of cartilage degradation. OA causes chronic pain because of the continuous inflammatory response and breakdown of joint cartilage, thus controlling 
the inflammatory response and cartilage degeneration is crucial for treatment (Alsalem et al. 2019).

In summary, PEA alleviates cartilage degradation and knee joint swelling in MIA-induced OA rats via a decrease in inflammatory mediators, such as $\mathrm{NO}, \mathrm{PGE}_{2}$, $\mathrm{LTB}_{4}, \mathrm{TNF}-\alpha$, and IL- $1 \beta$, and suppression of the secretion of cartilage-degrading enzymes, such as MMP-1, MMP3, MMP-9, and MMP-13. Thus, PEA may be a potential novel therapeutic drug for OA.

Acknowledgements We thank Gencor Pacific Ltd. for providing PEA.

Author contributions Conceptualization, HSL and EJK; methodology, JIJ, HSL, and EJK; formal analysis, JJJ, YEJ, SMK, and SHH; investigation, EJK; original draft preparation, HSL, JIJ, and EJK; writingreview and editing, HSL, EJK, JMM, CYL, and YHK.

Funding This study received no external funding.

Availability of data and materials The dataset generated during the present study is available upon reasonable request to the corresponding author (Eun Ji Kim).

Code availability Not applicable.

\section{Declarations}

Conflict of interest The authors declare no conflicts of interest.

Ethical approval All experiments using animals reported herein were performed according to protocols approved by the Institutional Animal Care and Use Committee of Hallym University (HallymR1 2018-75).

Consent to participate Not applicable.

Consent for publication Not applicable.

Open Access This article is licensed under a Creative Commons Attribution 4.0 International License, which permits use, sharing, adaptation, distribution and reproduction in any medium or format, as long as you give appropriate credit to the original author(s) and the source, provide a link to the Creative Commons licence, and indicate if changes were made. The images or other third party material in this article are included in the article's Creative Commons licence, unless indicated otherwise in a credit line to the material. If material is not included in the article's Creative Commons licence and your intended use is not permitted by statutory regulation or exceeds the permitted use, you will need to obtain permission directly from the copyright holder. To view a copy of this licence, visit http://creativecommons.org/licenses/by/4.0/.

\section{References}

Alhouayek M, Muccioli GG (2014) Harnessing the anti-inflammatory potential of palmitoylethanolamide. Drug Discov Today 19:1632 1639. https://doi.org/10.1016/j.drudis.2014.06.007

Alsalem M, Haddad M, Aldossary SA, Kalbouneh H, Altarifi A, Jaffal SM, Abbas MA, Aldaoud N, El-Salem K (2019) Role of cannabinoid receptor 1 and the peroxisome proliferator-activated receptor $\alpha$ in mediating anti-nociceptive effects of synthetic cannabinoids and a cannabinoid-like compound. Inflammopharmacology 27:1131-1142. https://doi.org/10.1007/s10787-019-00584-7

Alshami AM (2014) Knee osteoarthritis related pain: a narrative review of diagnosis and treatment. Int J Health Sci (qassim) 8:85-104. https://doi.org/10.12816/0006075

Audial S, Bonnotte B (2015) Inflammation. Rev Prat 65:403-408

Bjordal JM, Klovning A, Ljunggren AE, Slørdal L (2007) Short-term efficacy of pharmacotherapeutic interventions in osteoarthritic knee pain: a meta-analysis of randomised placebo-controlled trials. Eur J Pain 11:125-138. https://doi.org/10.1016/j.ejpain.2006. 02.013

Britti D, Crupi R, Impellizzeri D, Gugliandolo E, Fusco R, Schievano C, Morittu VM, Evangelista M, Di Paola R, Cuzzocrea S (2017) A novel composite formulation of palmitoylethanolamide and quercetin decreases inflammation and relieves pain in inflammatory and osteoarthritic pain models. BMC Vet Res 13:229. https:// doi.org/10.1186/s12917-017-1151-z

Chadjichristos C, Ghayor C, Kypriotou M, Martin G, Renard E, AlaKokko L, Suske G, de Crombrugghe B, Pujol JP, Galéra P (2003) $\mathrm{Sp} 1$ and $\mathrm{Sp} 3$ transcription factors mediate interleukin-1 beta down-regulation of human type II collagen gene expression in articular chondrocytes. J Biol Chem 278:39762-39772. https:// doi.org/10.1074/jbc.M303541200

Chen D, Shen J, Zhao W, Wang T, Han L, Hamilton JL, Im HJ (2017) Osteoarthritis: toward a comprehensive understanding of pathological mechanism. Bone Res 5:16044. https://doi.org/10.1038/ boneres. 2016.44

Clockaerts S, Bastiaansen-Jenniskens YM, Feijt C, Verhaar JA, Somville J, De Clerck LS, Van Osch GJ (2011) Peroxisome proliferator activated receptor alpha activation decreases inflammatory and destructive responses in osteoarthritic cartilage. Osteoarthritis Cartilage 19:895-902. https://doi.org/10.1016/j.joca.2011.03.010

Costa B, Comelli F, Bettoni I, Colleoni M, Giagnoni G (2008) The endogenous fatty acid amide, palmitoylethanolamide, has antiallodynic and anti-hyperalgesic effects in a murine model of neuropathic pain: involvement of $\mathrm{CB}(1)$, TRPV1 and PPARgamma receptors and neurotrophic factors. Pain 139:541-550. https://doi. org/10.1016/j.pain.2008.06.003

Darmani NA, Izzo AA, Degenhardt B, Valenti M, Scaglione G, Capasso R, Sorrentini I, Di Marzo V (2005) Involvement of the cannabimimetic compound, $\mathrm{N}$-palmitoyl-ethanolamine, in inflammatory and neuropathic conditions: review of the available pre-clinical data, and first human studies. Neuropharmacology 48:1154-1163. https://doi.org/10.1016/j.neuropharm.2005.01.001

D'Souza WN, Ng GY, Youngblood BD, Tsuji W, Lehto SG (2011) A review of current animal models of osteoarthritis pain. Curr Pharm Biotechnol 12:1596-1612. https://doi.org/10.2174/13892 0111798357320

Fahmi H, Di Battista JA, Pelletier JP, Mineau F, Ranger P, MartelPelletier J (2001) Peroxisome proliferator-activated receptor gamma activators inhibit interleukin-1beta-induced nitric oxide and matrix metalloproteinase 13 production in human chondrocytes. Arthritis Rheum 44:595-607. https://doi.org/10.1002/15290131(200103)44:3\%3c595::AID-ANR108\%3e3.0.CO;2-8

Fusco M, Skaper SD, Coaccioli S, Varrassi G, Paladini A (2017) Degenerative joint diseases and neuroinflammation. Pain Pract 17:522-532. https://doi.org/10.1111/papr.12551

Gatti A, Lazzari M, Gianfelice V, Di Paolo A, Sabato E, Sabato AF (2012) Palmitoylethanolamide in the treatment of chronic pain caused by different etiopathogenesis. Pain Med 13:1121-1130. https://doi.org/10.1111/j.1526-4637.2012.01432.x

Glasson SS, Chambers MG, van Den Berg WB, Little CB (2010) The OARSI histopathology initiative - recommendations for histological assessments of osteoarthritis in the mouse. Osteoarthr Cartil 18:S17-S23. https://doi.org/10.1016/j.joca.2010.05.025 
Guida F, Luongo L, Boccella S et al (2017) Palmitoylethanolamide induces microglia changes associated with increased migration and phagocytic activity: involvement of the CB2 receptor. Sci Rep 7:375. https://doi.org/10.1038/s41598-017-00342-1

Hesselink JM, Hekker TA (2012) Therapeutic utility of palmitoylethanolamide in the treatment of neuropathic pain associated with various pathological conditions: a case series. J Pain Res 5:437-442. https://doi.org/10.2147/JPR.S32143

Hinz B, Brune K (2004) Pain and osteoarthritis: new drugs and mechanisms. Curr Opin Rheumatol 16:628-633. https://doi.org/10.1097/ 01.hco.0000136130.95746.14

Hochman JR, French MR, Bermingham SL, Hawker GA (2010) The nerve of osteoarthritis pain. Arthritis Care Res (hoboken) 62:1019-1023. https://doi.org/10.1002/acr.20142

Huang D, Zhao Q, Liu H, Guo Y, Xu H (2016) PPAR- $\alpha$ Agonist WY-14643 Inhibits LPS-Induced Inflammation in Synovial Fibroblasts via NF-kB Pathway. J Mol Neurosci 59:544-553. https:// doi.org/10.1007/s12031-016-0775-y

Impellizzeri D, Esposito E, Di Paola R, Ahmad A, Campolo M, Peli A, Morittu VM, Britti D, Cuzzocrea S (2013) Palmitoylethanolamide and luteolin ameliorate development of arthritis caused by injection of collagen type II in mice. Arthritis Res Ther 15:R192. https://doi.org/10.1186/ar4382

Kuyinu EL, Narayanan G, Nair LS, Laurencin CT (2016) Animal models of osteoarthritis: classification, update, and measurement of outcomes. J Orthop Surg Res 11:19. https://doi.org/10.1186/ s13018-016-0346-5

La Porta C, Bura SA, Negrete R, Maldonado R (2014) Involvement of the endocannabinoid system in osteoarthritis pain. Eur J Neurosci 39:485-500. https://doi.org/10.1111/ejn.12468

LoVerme J, La Rana G, Russo R, Calignano A, Piomelli D (2005) The search for the palmitoylethanolamide receptor. Life Sci 77:16851698. https://doi.org/10.1016/j.lfs.2005.05.012

Man GS, Mologhianu G (2014) Osteoarthritis pathogenesis: a complex process that involves the entire joint. J Med Life 7:37-41

Maniar KH, Jones IA, Gopalakrishna R, Vangsness CT (2018) Lowering side effects of NSAID usage in osteoarthritis: recent attempts at minimizing dosage. Expert Opin Pharmacother 19:93-102. https://doi.org/10.1080/14656566.2017.1414802

Mora JC, Przkora R, Cruz-Almeida Y (2018) Knee osteoarthritis: pathophysiology and current treatment modalities. J Pain Res 11:2189-2196. https://doi.org/10.2147/JPR.S154002

Paladini A, Fusco M, Cenacchi T, Schievano C, Piroli A, Varrassi G (2016) Palmitoylethanolamide, a special food for medical purposes, in the treatment of chronic pain: a pooled data meta-analysis. Pain Physician 19:11-24

Petrosino S, Di Marzo V (2017) The pharmacology of palmitoylethanolamide and first data on the therapeutic efficacy of some of its new formulations. Br J Pharmacol 174:1349-1365. https://doi. org/10.1111/bph.13580

Pritzker KP, Gay S, Jimenez SA, Ostergaard K, Pelletier JP, Revell PA, Salter D, van den Berg WB (2006) Osteoarthritis cartilage histopathology: grading and staging. Osteoarthr Cartil 14:13-29. https://doi.org/10.1016/j.joca.2005.07.014
Re G, Barbero R, Miolo A, Di Marzo V (2007) Palmitoylethanolamide, endocannabinoids and related cannabimimetic compounds in protection against tissue inflammation and pain: potential use in companion animals. Vet J 173:21-30. https://doi.org/10.1016/j. tvj1.2005.10.003

Richardson D, Pearson RG, Kurian N, Latif ML, Garle MJ, Barrett DA, Kendall DA, Scammell BE, Reeve AJ, Chapman V (2008) Characterisation of the cannabinoid receptor system in synovial tissue and fluid in patients with osteoarthritis and rheumatoid arthritis. Arthritis Res Ther 10:R43. https://doi.org/10.1186/ar2401

Rontein D, Nishida I, Tashiro G, Yoshioka K, Wu WI, Voelker DR, Basset G, Hanson AD (2001) Plants synthesize ethanolamine by direct decarboxylation of serine using a pyridoxal phosphate enzyme. J Biol Chem 276:35523-35529. https://doi.org/10.1074/ jbc.M106038200

Sagar DR, Staniaszek LE, Okine BN et al (2010) Tonic modulation of spinal hyperexcitability by the endocannabinoid receptor system in a rat model of osteoarthritis pain. Arthritis Rheum 62:36663676. https://doi.org/10.1002/art.27698

Saklatvala J (1986) Tumour necrosis factor alpha stimulates resorption and inhibits synthesis of proteoglycan in cartilage. Nature 322:547-549. https://doi.org/10.1038/322547a0

Scott E, Peter F, Sanders J (2007) Biomass in the manufacture of industrial products-the use of proteins and amino acids. Appl Microbiol Biotechnol 75:751-762. https://doi.org/10.1007/ s00253-007-0932-x

Steels E, Venkatesh R, Steels E, Vitetta G, Vitetta L (2019) A doubleblind randomized placebo controlled study assessing safety, tolerability and efficacy of palmitoylethanolamide for symptoms of knee osteoarthritis. Inflammopharmacology 27:475-485. https:// doi.org/10.1007/s10787-019-00582-9

Trumble TN (2005) The use of nutraceuticals for osteoarthritis in horses. Vet Clin North Am Equine Pract 21(575-97):v-vi. https:// doi.org/10.1016/j.cveq.2005.08.004

Udo M, Muneta T, Tsuji K, Ozeki N, Nakagawa Y, Ohara T, Saito R, Yanagisawa K, Koga H, Sekiya I (2016) Monoiodoacetic acid induces arthritis and synovitis in rats in a dose- and time-dependent manner: proposed model-specific scoring systems. Osteoarthr Cartil 24:1284-1291. https://doi.org/10.1016/j.joca.2016.02.005

Wang J, Wang G, Sun GW (2016) Role of PPAR $\alpha$ in down-regulating AGE-induced TGF- $\beta$ and MMP-9 expressions in chondrocytes. Genet Mol Res. https://doi.org/10.4238/gmr.15027963

Zhang W, Ouyang H, Dass CR, Xu J (2016) Current research on pharmacologic and regenerative therapies for osteoarthritis. Bone Res 4:15040. https://doi.org/10.1038/boneres.2015.40

Publisher's Note Springer Nature remains neutral with regard to jurisdictional claims in published maps and institutional affiliations. 